\title{
Genetic analysis of the Trichuris muris-induced model of colitis reveals QTL overlap and a novel gene cluster for establishing colonic inflammation
}

Scott E Levison ${ }^{1}$, Paul Fisher ${ }^{2}$, Jenny Hankinson ${ }^{3}$, Leo Zeef ${ }^{4}$, Steve Eyre ${ }^{5}$, William E Ollier ${ }^{3}$, John T McLaughlin ${ }^{1}$, Andy Brass ${ }^{6}$, Richard K Grencis ${ }^{7 \dagger}$ and Joanne L Pennock ${ }^{1 *+}$

\begin{abstract}
Background: Genetic susceptibility to colonic inflammation is poorly defined at the gene level. Although Genome Wide Association studies (GWAS) have identified loci in the human genome which confer susceptibility to Inflammatory Bowel Disease (Crohn's and Ulcerative Colitis), it is not clear if precise loci exist which confer susceptibility to inflammation at specific locations within the gut e.g. small versus large intestine. Susceptibility loci for colitis in particular have been defined in the mouse, although specific candidate genes have not been identified to date. We have previously shown that infection with Trichuris muris (T. muris) induces chronic colitis in susceptible mouse strains with clinical, histological, and immunological homology to human colonic Crohn's disease. We performed an integrative analysis of colitis susceptibility, using an F2 inter-cross of resistant (BALB/C) and susceptible (AKR) mice following T. muris infection. Quantitative Trait Loci (QTL), polymorphic and expression data were analysed alongside in silico workflow analyses to discover novel candidate genes central to the development and biology of chronic colitis.

Results: 7 autosomal QTL regions were associated with the establishment of chronic colitis following infection. 144 QTL genes had parental strain SNPs and significant gene expression changes in chronic colitis (expression fold-change $\geq+/-1.4$ ). The T. muris QTL on chromosome 3 (Tm3) mapped to published QTL in 3 unrelated experimental models of colitis and contained 33 significantly transcribed polymorphic genes. Phenotypic pathway analysis, text mining and time-course GPCR replication highlighted several potential cis-QTL candidate genes in colitis susceptibility, including FcgR1, Ptpn22, RORC, and Vav3.
\end{abstract}

Conclusion: Genetic susceptibility to induced colonic mucosal inflammation in the mouse is conserved at $\operatorname{Tm} 3$ and overlays Cdcs 1.1. Genes central to the maintenance of intestinal homeostasis reside within this locus, implicating several candidates in susceptibility to colonic inflammation. Combined methodology incorporating genetic, transcriptional and pathway data allowed identification of biologically relevant candidate genes, with Vav3 newly implicated as a colitis susceptibility gene of functional relevance.

Keywords: Trichuris muris, Colitis, Genetic susceptibility, Cdcs1, Crohn's

\footnotetext{
* Correspondence: Joanne.pennock@manchester.ac.uk

${ }^{\dagger}$ Equal contributors

${ }^{1}$ Gastrointestinal Sciences, Institute of Inflammation and Repair, Faculty of

Medicine and Human Sciences, University of Manchester, 4.004 AV Hill

Building, Oxford Road, Manchester M13 9PT, UK

Full list of author information is available at the end of the article
} 


\section{Background}

Many diseases result from the complex interaction of environmental and genetic factors (e.g. Crohn's disease, diabetes mellitus) [1,2]. Phenotypic expression is influenced by multiple genes, which individually may increase or decrease the probability of disease development. Gene variation and gene-gene interactions, additionally results in non-linear contributions to phenotypic variation. Discovering the genetic architecture of complex traits thus represents a true challenge [3] and requires collaborative multi-disciplinary investigation and a variety of experimental approaches $[4,5]$. The exploration of new animal models of colitis with well-defined phenotypes and homology to human pathology, provide a comparative approach to refine biological discoveries for subsequent human translation.

Trichuris muris, a natural intestinal parasite of mice has been extensively studied as a model for human whipworm (Trichuris trichiura) infection. In dissecting the immune response to Trichuris infection, a paradigm of resistance and susceptibility to chronic colonic inflammation has emerged [6]. Following the ingestion of parasite ova, acute colitis develops in all mice, but it is the genetic composition of mouse strain which dictates the presence of colitis. BALB/c mice mount immunemediated $\mathrm{T}_{\mathrm{H}} 2$ dependent parasite expulsion (IL4, IL5 and IL13 expression) [6,7] with full resolution within 20 days. Conversely AKR mice sustain a chronic Trichuris infection, respond with a $\mathrm{T}_{\mathrm{H}} 1$ immune response (IFN $\gamma$, IL12), and subsequent establishment of colitis [8]. These polarized outcomes occur despite identical treatment and conditioning, and are almost certainly determined by host genetic variation. Importantly, we have recently characterised differences in colonic tissue transcription between susceptible and resistant mice and demonstrated phenotypic, immunological and biological pathway homology to human Crohn's disease [9]. These data present T. muris infection not as an aetiological factor in the pathogenesis of Crohn's disease, nor solely a model of infection but as a viable and relevant colitis model to investigate and study mucosal inflammation.

The multifactorial and complex nature of Crohn's disease remains to be fully characterised, but it is evident that disease can be initiated anywhere along the digestive tract. It is likely that precise environmental triggers determine the site of initiation, but it is also possible that host genetics play a part. A variety of experimental models have been developed to study pathogenic mechanisms responsible for the induction and perpetuation of Crohn's disease. Phenotypic and biological factors common between colonic Crohn's disease and chronic T. muris induced colitis, present a novel opportunity to characterise the genetic architecture central to disease susceptibility in the colon. The aim of the current study was to identify genome wide genetic elements and mechanistic pathways which underpin the development and maintenance of such chronic inflammation.

\section{Results}

Systemic and colonic phenotyping of chronic T. muris colitis in an F2 population of resistant and susceptible mice

An F2 inter-cross of resistant (BALB/c) and susceptible (AKR) mice was phenotyped 35 days post-infection, a time-point when chronic inflammation is established in susceptible mice (Figure 1). Colonic worm burden was not normally distributed; the majority of animals were resistant, with the largest worm burdens harboured by a small number of individuals (Figure 1A). This pattern of worm load distribution is indicative of an out-bred cohort [10]. Serum parasite-specific IgG1 ( $\mathrm{T}_{\mathrm{H}} 2$ specific) and IgG2a ( $\mathrm{T}_{\mathrm{H}} 1$ specific) was measured. Many individuals had a combination of both serotypes. To determine the predominant phenotype expressed a serum IgG1:2a ratio was calculated. A highly significant difference was observed between the mean of IgG1:2a ratio in resistant and susceptible mice (Mann Whitney $\mathrm{U}$ test, $\mathrm{p}<0.0001$, Figure 1B). At day 35 post-infection, 83\% (110/133) of individuals with persistent worm burden demonstrated serum antibody titre IgG1 < IgG2a, indicative of a polarised $\mathrm{T}_{\mathrm{H}} 1$ immune response. A dominant $\mathrm{T}_{\mathrm{H}} 2$ immune response (IgG1 > IgG2a) correlated with worm expulsion. Furthermore, females demonstrated more resistance compared to males; females had significantly fewer worms at D35 post infection (Additional file 1: Figure S1A) and significantly higher IgG1:2a ratio $(\mathrm{p}<0.0001$, Additional file 1: Figure $\mathrm{S} 1 \mathrm{~B}$ ) indicative of a dominant Th2 immune response.

Colonic histological assessment demonstrated persistent $T$. muris infection and large bowel inflammation. Mild-to-moderate inflammatory changes included: transmural tissue oedema and associated leukocytic infiltration (lymphocytes, macrophages, neutrophils); prominent mucosal and submucosal reactive lymphoid aggregates; colonic crypt hyperplasia and hypertrophy (Figure 1C-E). Significant correlation between histological parameters of inflammation (e.g. crypt length), immune response phenotype (Figure 1F: Spearman's Rs $=-0.54$ ) and worm burden (Figure 1G: Spearman's Rs $=0.84$ ), were demonstrated. 98.5\% of mice with persistent helminthosis demonstrated colonic inflammatory changes.

\section{Whole genome Linkage analysis}

In total 7 QTL demonstrated significant correlation between susceptibility phenotype and genotype. Chromosomal locus, LOD scoring, trait correlation and the number of genes found within each QTL were defined (Table 1). The majority of QTL were associated with both a $\mathrm{T}_{\mathrm{H}} 1$ pro-inflammatory immune response, as 


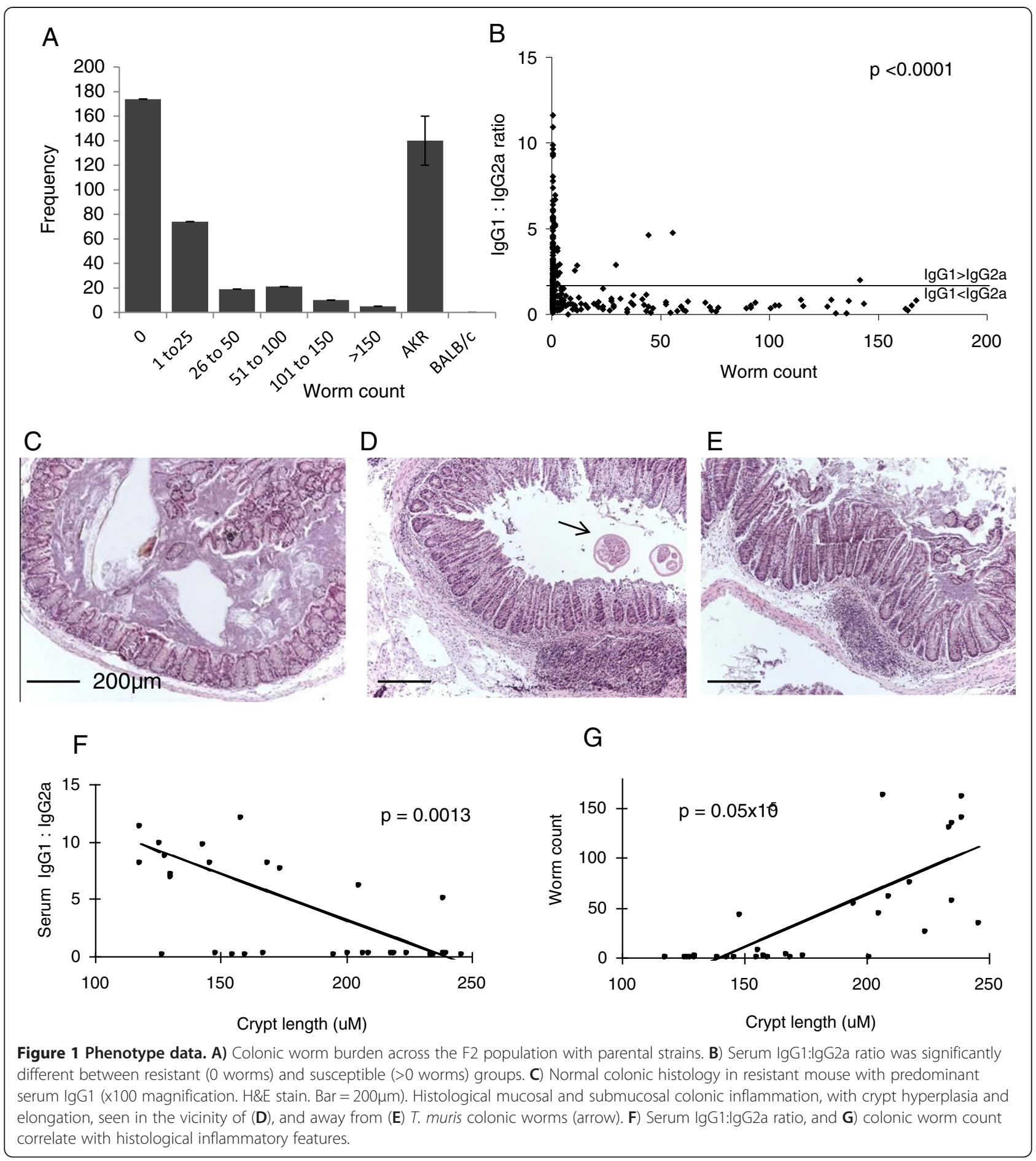

reflected in low IgG1:IgG2a ratio, and persistent worm burden. Tm10 however, was solely indicative of continued worm persistence. Tm17 demonstrated the most significant LOD score, overlying the major histocompatibility complex (MHC).

Of particular interest, Tm3 (92.4-118.3 Mbp, chromosome 3) demonstrated complete overlap with a sus- ceptibility locus identified in three unrelated murine models of spontaneous experimental colitis: G-protein alpha inhibitory 2 chain knock-out $\left(\right.$ Gnai2 $\left.^{--}\right)$mice (Gpdc1 locus) [11]; C3H/HeJBir IL10-deficient mice (Cdcs1 locus) [12]; T-bet ${ }^{-1}$ Rag2 $^{-/-}$double-deficient mice that resemble ulcerative colitis (TRUC) (Cdcs1 locus) [13]. The Cdcs1 region has been shown to contain at least three distinct 
Table 1 Summary of Trichuris muris QTL (Tm) found across the genome

\begin{tabular}{|c|c|c|c|c|c|c|c|c|}
\hline $\begin{array}{l}\text { T. Muris } \\
\text { QTL }\end{array}$ & Murine chrom & Left marker & $\begin{array}{c}\text { Right } \\
\text { marker }\end{array}$ & $\operatorname{LOD}^{\mathrm{a}}$ & Trait & Dominance & $\begin{array}{l}\text { Gene no. within } \\
\text { QTL (Ensembl) }\end{array}$ & $\begin{array}{c}\text { AKR vs BALB/c } \\
\text { genes with SNPs }\end{array}$ \\
\hline $\operatorname{Tm} 1$ & 1 & D1Mit33 & D1Mit36 & 2.39 & Serum IgG's Worm count & -8.3 & 90 & 68 \\
\hline $\operatorname{Tm} 3$ & 3 & $\begin{array}{l}\text { D3Mit156 } \\
\text { 92.4Mbp }\end{array}$ & $\begin{array}{l}\text { D3Mit79 } \\
\text { 118.3Mbp }\end{array}$ & 2.80 & Serum IgG's Worm count & 8.4 & 342 & 270 \\
\hline Tm4 & 4 & D4Mit166 & D4Mit12 & 3.23 & Serum lgG's & -5.3 & 311 & 289 \\
\hline $\operatorname{Tm} 10$ & 10 & D10Mit14 & D10Mit35 & 4.10 & Worm count & - & 27 & 24 \\
\hline $\operatorname{Tm} 11$ & 11 & D11Mit99 & D11Mit61 & 4.14 & Serum IgG's Worm count & -0.3 & 265 & 182 \\
\hline $\operatorname{Tm} 12$ & 12 & D12Mit285 & D12Mit201 & 4.7 & Serum IgG's Worm count & -10.7 & 95 & 75 \\
\hline $\operatorname{Tm} 17$ & 17 & D17Mit175 & D17Mit176 & 8.04 & Serum lgG's Worm count & -5.7 & 287 & 251 \\
\hline
\end{tabular}

${ }^{\mathrm{a}}$ For non-parametric traits, Kruskal Wallis values were converted to LOD according to published methods [13].

regions $[14,15]$. Here, we show complete overlap of Tm3 with Cdcs1.1 (Figure 2), a region shown to contribute strongly to the severity of colitis in $\mathrm{C} 3 \mathrm{H} / \mathrm{HeJBir}$ mice $[14,15]$.

\section{Prioritization of QTL candidate genes via pathway-driven workflow analysis}

Schematic representation and key stage data from each of the qtl_to_pathway [16] and refseq_ids_to_pathways [17] workflows are shown (Figure 3). In total, 1419 genes were identified within the $7 \mathrm{~T}$. muris QTL. Genes attributed to one or more KEGG biological pathways were determined (Figure 3.1). Simultaneously, of 5476 genes with significant transcriptional differences during T. muris infection [9], 2504 genes displayed either an up-regulated or down-regulated change in expression of $\geq 1.4$ fold (i.e. a $40 \%$ or greater change in expression over naïve controls, Figure 3.2). Biological KEGG pathways associated with significant gene expression data were determined. In total, 1158 of these genes (46\%) were involved in 204 separate KEGG pathways (Figure 3.3).

The cross correlation of functional pathways containing QTL genes and genes demonstrating significant expression were identified, linking genotype and phenotype trait interactions (Figure 3.3). Finally, polymorphic genes between parental AKR and BALB/c mice were identified within each locus (Figure 3.4).

As an example, 344 Ensembl gene ID's were detected within Tm3. Of these, 97 (28\%) were designated as functionally important within molecular interaction networks, as assigned by the KEGG pathway database. Significantly

Cdcs 1.1

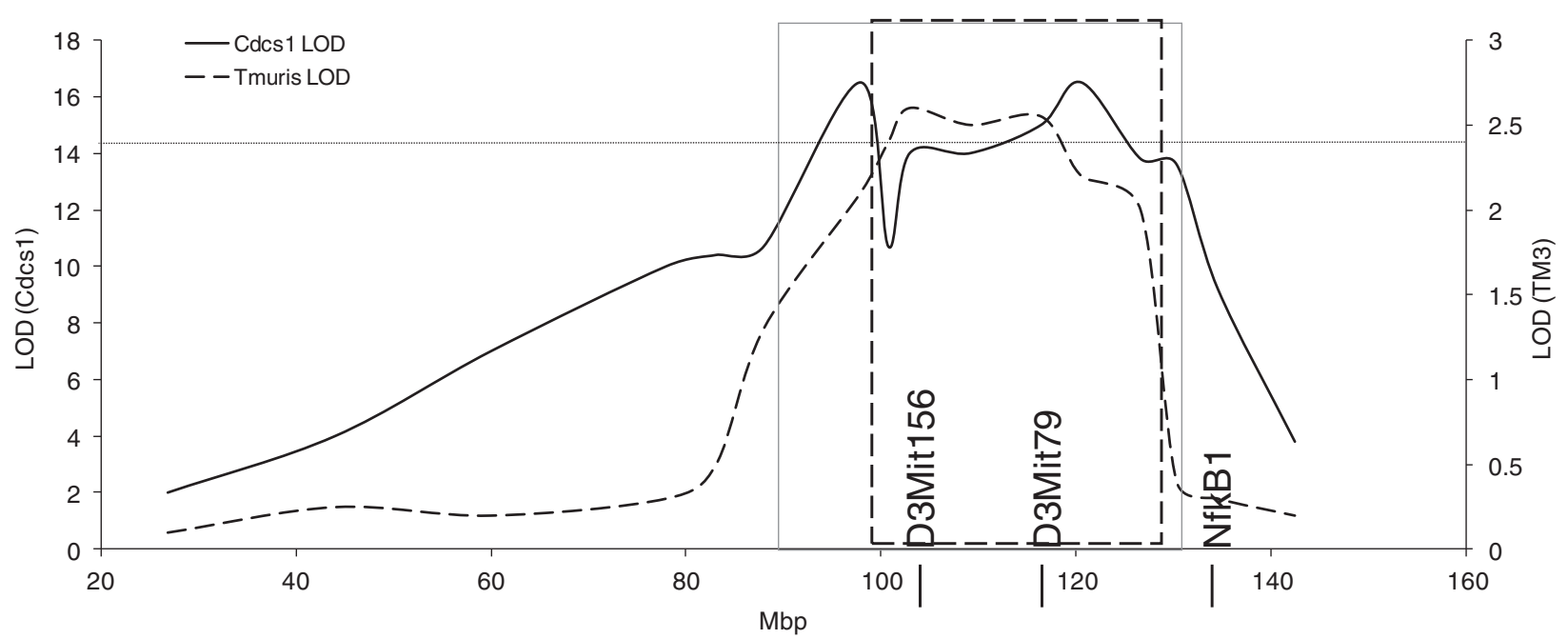

Figure 2 Tm3 overlays the colitic Cdcs1 QTL. Previous congenic analysis defined Cdcs1 between 87.1 and 131.1 Mbp (solid box), and overlays gpdc1 (dashed box), a mouse QTL which also correlates with spontaneous colitis. T. muris QTL Tm3 (broken line) lies between D3Mit156 (92 Mbp) and D3Mit79 (118 Mbp), outside the location of a previously defined candidate gene NfkB1 (135.1 Mbp). The threshold for suggestive correlation is shown for Tm3 at LOD 2.4 


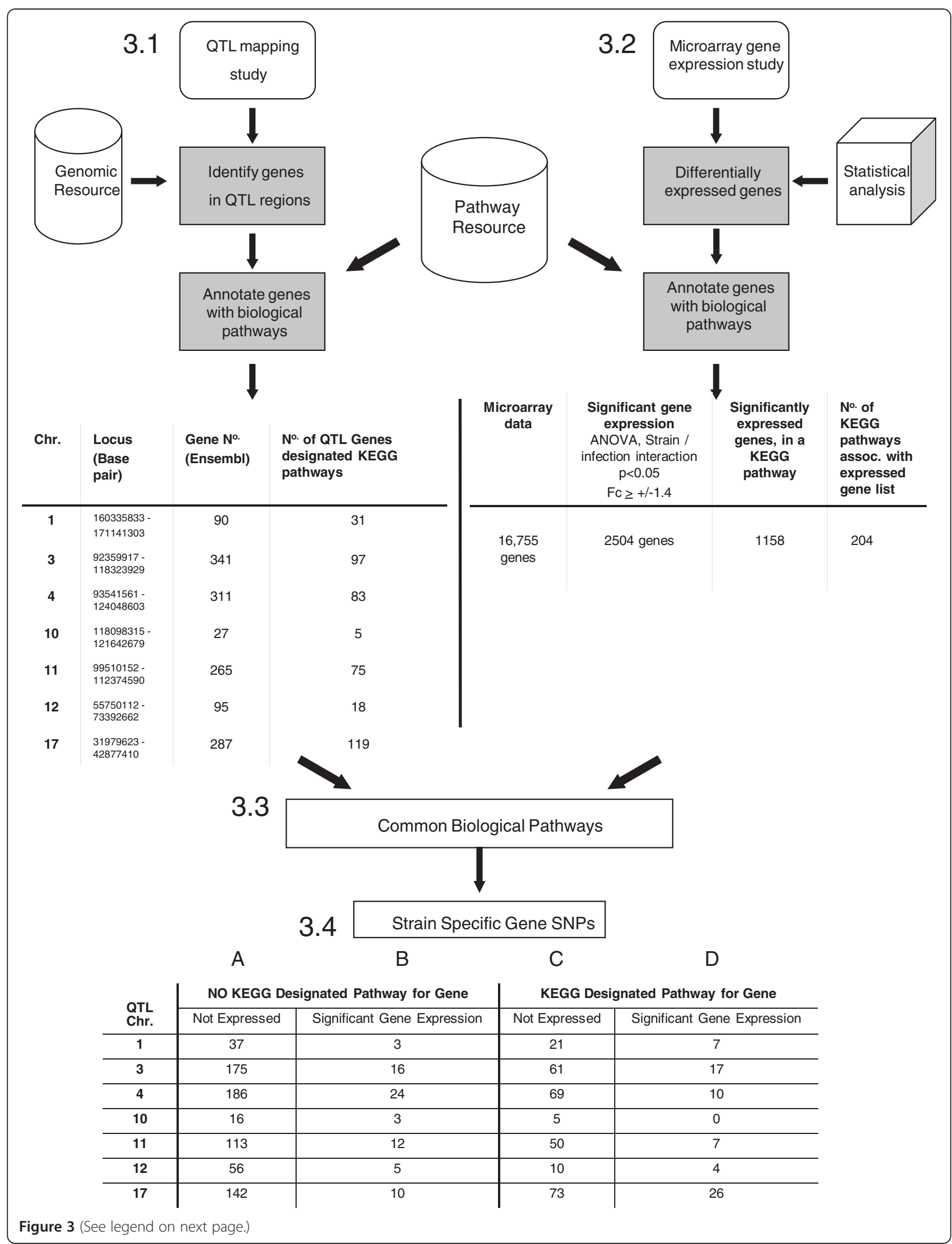


(See figure on previous page.)

Figure 3 Flow diagram showing unbiased identification of candidate genes in identified QTL. 3.1: Genes within QTL were identified and assigned biological pathways. 3.2: In parallel, genes in QTL with different relative expression between parental strains were assigned biological pathways. 3.3 \& 3.4: Genes within commonly identified pathways were ranked according to SNP number (AKR vs BALB/c www.sanger.ac.uk).

expressed microarray genes were similarly assigned biological pathways. For Tm3, the cross correlation of common pathway data and the exclusion of any gene which lacked SNPs between parental strains, identified 17 Quantitative Trait genes (Figure 3.4, Column D). In comparison, 61 KEGG-assigned polymorphic genes did not demonstrate any change in transcriptional activity (Column C). Of the genes yet to be allocated a KEGG pathway, 16 of 191 genes displayed significant transcription (Column B). The same process was undertaken for all 7 QTL (Figure 3).

\section{Chromosome 3 candidates}

Analysis of $T m 3$, revealed 33 polymorphic genes with significant transcriptional changes during infection. Candidate genes were analysed in two distinct subsets; those with and those without a designated KEGG pathway. Of the 33 genes, 17 demonstrated a central mechanistic role within one or more KEGG pathway (Table 2). Vav3 was associated with the highest number of pathways $(n=7)$. Candidate genes were ranked according to the number of SNPs that occurred between AKR and BALB/c strains. Vav3 was the gene with the highest number of SNP variants $(n=2047)$.

The 16 genes with no KEGG pathway association were subsequently analysed using a workflow-based text-mining approach, to allow prioritization according to known biological roles in inflammation or gut immunology. Genes were ranked according to a cosine vector score (see Methods), estimating the significance of correlation between candidate gene and phenotype. SNP variation

Table 2 Significantly expressed Tm3 genes possessing strain-specific SNPs and a designated biological (KEGG) pathway

\begin{tabular}{|c|c|c|c|c|c|}
\hline Gene & KEGG designated pathway & SNP no. & Gene & KEGG designated pathway & SNP no. \\
\hline \multirow[t]{7}{*}{ Vav3 } & Chemokine signalling pathway & 2047 & Fcgr1 & Fc gamma R-mediated phagocytosis & 18 \\
\hline & Fc gamma R-mediated phagocytosis & & & Hematopoietic cell lineage & \\
\hline & Focal adhesion & & & Leishmaniasis & \\
\hline & Leukocyte transendothelial migration & & & Phagosome & \\
\hline & T cell receptor signalling pathway & & & Systemic lupus erythematosus & \\
\hline & B cell receptor signalling pathway & & Prpf38b & Spliceosome & 9 \\
\hline & Regulation of actin cytoskeleton & & & & \\
\hline \multirow[t]{5}{*}{ Hmgcs2 } & Butanoate metabolism & 134 & Vcam1 & Cell adhesion moleculaes (CAMs) & 8 \\
\hline & Metabolic pathways & & & Leukocyte transendothelial migration & \\
\hline & Synthesis and degradation of ketone & & & Malaria & \\
\hline & Terpenoid backbone biosynthesis & & Cdc14a & Cell cycle & 7 \\
\hline & Valine, leuckine and isoleucine degradation & & & & \\
\hline \multirow[t]{3}{*}{ Ctss } & Antigen processing and presentation & 120 & Dbt & Metabolic pathways & 2 \\
\hline & Lysosome & & & Valine, leucine and isoleucine degradation & \\
\hline & Phagosome & & & & \\
\hline Ap4b1 & Lysosome & 92 & Gstm7 & Drug metabolism - cytochrome P450 & 2 \\
\hline \multirow[t]{2}{*}{ Rorc } & Circadian rhythm & 76 & & Glutathione metabolism & \\
\hline & & & & Metabolism of xenobiotics by cytochrome p450 & \\
\hline \multirow[t]{3}{*}{ Gstm3 } & Drug metabolism - cytochrome P450 & 73 & Hsd3b6 & Metabolic pathways & 2 \\
\hline & Glutathione metabolism & & & Steroid hormone biosynthesis & \\
\hline & Metabolism of xenobiotcis by cytochrome P450 & & & & \\
\hline \multirow[t]{5}{*}{ Dpyd } & Beta-Alanin metabolism & 24 & Fmo5 & Drug metabolism - cytochrome p450 & 1 \\
\hline & Drug metabolism - other enzymes & & & & \\
\hline & Metabolic pathways & & Gstm6 & Drug metabolism - cytochrome p450 & 1 \\
\hline & Pantothenate and CoA biosynthesis & & & & \\
\hline & Pyrimidine metabolism & & Hist2h2be & Systemic lupus erythematosus & 1 \\
\hline
\end{tabular}


between parental strains was also considered. As a result, additional proposed candidates included: Ptpn22, S100a10, and Slc22a15 (Table 3).

\section{Candidate gene validation}

Quantitative PCR analysis was undertaken independently in infected parental strains (days $0,7,14,21$, and 35 postinfection) to validate microarray data (Tables 2 and 3).

For gene candidates, Vav3, Ptpn22, FcgR1 and S100a10, qPCR corroborated up-regulated expression found in susceptible AKR on day 35 post infection (Figure 4). Likewise, the down-regulation of Hmgcs 2 was confirmed by qPCR. Microarray analysis of Ctss and RORc demonstrated down regulation of colonic gene expression in chronically affected individuals, which could not be replicated (data not shown for $R O R c$ ).

\section{Discussion}

Trichuris muris-induced colitis represents a tractable murine model for understanding the patho-biological mechanisms of chronic intestinal inflammation $[9,19]$. The use of Quantitative Trait Loci (QTL) mapping based on continuous phenotypic variation has proved a useful technique in many murine polygenic traits including intestinal inflammation [12,20,21]. Yet, of more than 2000

Table 3 Significantly expressed genes possessing strainspecific SNPs but as of yet, undesignated a biological (KEGG) pathway

\begin{tabular}{lcc}
\hline Gene & Text mining (cosine vector score) & SNP no. \\
\hline S100a10 & $\mathbf{0 . 2 4 8}$ & $\mathbf{6 2}$ \\
Slc22a150 & 0.0219 & 19 \\
Selenbp1 & 0.0065 & 19 \\
Mov10 & 0.0063 & 3 \\
Ppm1j & 0.0013 & 3 \\
Pogz & 0.0009 & 8 \\
Extl2 & 0.0008 & 2 \\
lgsf3 & 0.0007 & 177 \\
Ptpn22 & 0.0006 & 276 \\
Selenbp2 & 0.0004 & 21 \\
Wdr & 0.0004 & 1 \\
Cd53 & 0.0002 & 92 \\
Cttnbp2nl & 0 & 55 \\
Golph31 & 0 & 34 \\
4933421E11Rsk & 0 & 19 \\
Eps8l3 & 0 & 1
\end{tabular}

Genes ranked according to text-mining significance, and SNP number for each gene noted. (QTL Chromosome 3, TM3). The top candidate is highlighted in bold: S100a10 had the highest cosine vector score demonstrating maximum relevance in the literature.

Transcript profiling: Microarray data has been submitted to ArrayExpress. Accession number E-MEXP-3098.
QTL documented within the mouse genome database [22] fewer than $1 \%$ of studies have actually been characterized at a gene or molecular level, due to the small effect size of the susceptibility locus in question $(<10 \%$ penetrance), or the large interval size defined [22]. New multi-factorial approaches have been discussed in the literature [23] and demonstrate that understanding complex genetic traits requires an integrative analysis.

Specific steps were taken in our experimental design to consider recent reports concerning the QTL/microarray approach in the identification of QTL candidate genes [23]. First, QTL were defined with regards to experimental phenotype (pQTL), and correlated with transcriptional expression activity in parental strains. Second, the use of high density Affymetrix exon array, which targets approximately 40 exonic probes per gene, overcame any problem of potential allelic-biased probe binding. Third, a hypothesis-free pathway analysis, backed up by additional text-mining, was employed in the secondary filtering of potential candidate genes to reduce bias. Fourth, any genes lacking polymorphisms (coding and non-coding) between parental strains were excluded from analyses, and lastly, positional overlap with a previously replicated major colitis susceptibility quantitative trait locus (Cdcs1) prioritised Tm3 for targeted analysis.

With regards to this shared locus, Cdcs1 on chromosome 3 was first noted in a QTL study of spontaneous colitis using IL10 deficient mice [12]. This locus has been shown to contain at least 3 distinct regions (Cdcs1.1, $1.2 \& 1.3)$ that contribute to a severe colitic phenotype $[14,15]$. Interestingly, all three regions contribute to caecal and proximal colonic inflammation strongly suggesting that this locus is a colitis 'hotspot' for susceptibility and/or regulation. Here we show complete overlap of Tm3 with Cdcs1.1 (Figure 2). Although $N F-k B 1$ has been suggested as a candidate gene for the Cdcs1 locus, it is clear that it is not responsible entirely for the severe pathology observed [15]. To date, FcgR1 remains the key candidate gene described in the Cdcs1.1 region $[14,15]$ and is corroborated by our findings. Additional association with colitis susceptibility in Gnai2 $2^{-/-}$ mice [11] suggests that this locus may govern key inflammatory pathways in disease development, irrespective of trigger. QTL mapping specifically highlighted the Cdcs1.3 region in the spontaneous colitis and colorectal cancer development of TRUC mice [13]. However, more distal colonic disease (distal third of the colon) or the potential for malignant transformation may not be represented at this sub-locus.

We have shown that at least 6 biologically significant and polymorphic candidate genes lie within the $C d c s 1.1$ autosomal region. Importantly, 4 of these candidate genes are key in pathways relevant in the context of human Crohn's disease (FcgR1, Vav3, Vcam1 and Ctss), a disease 

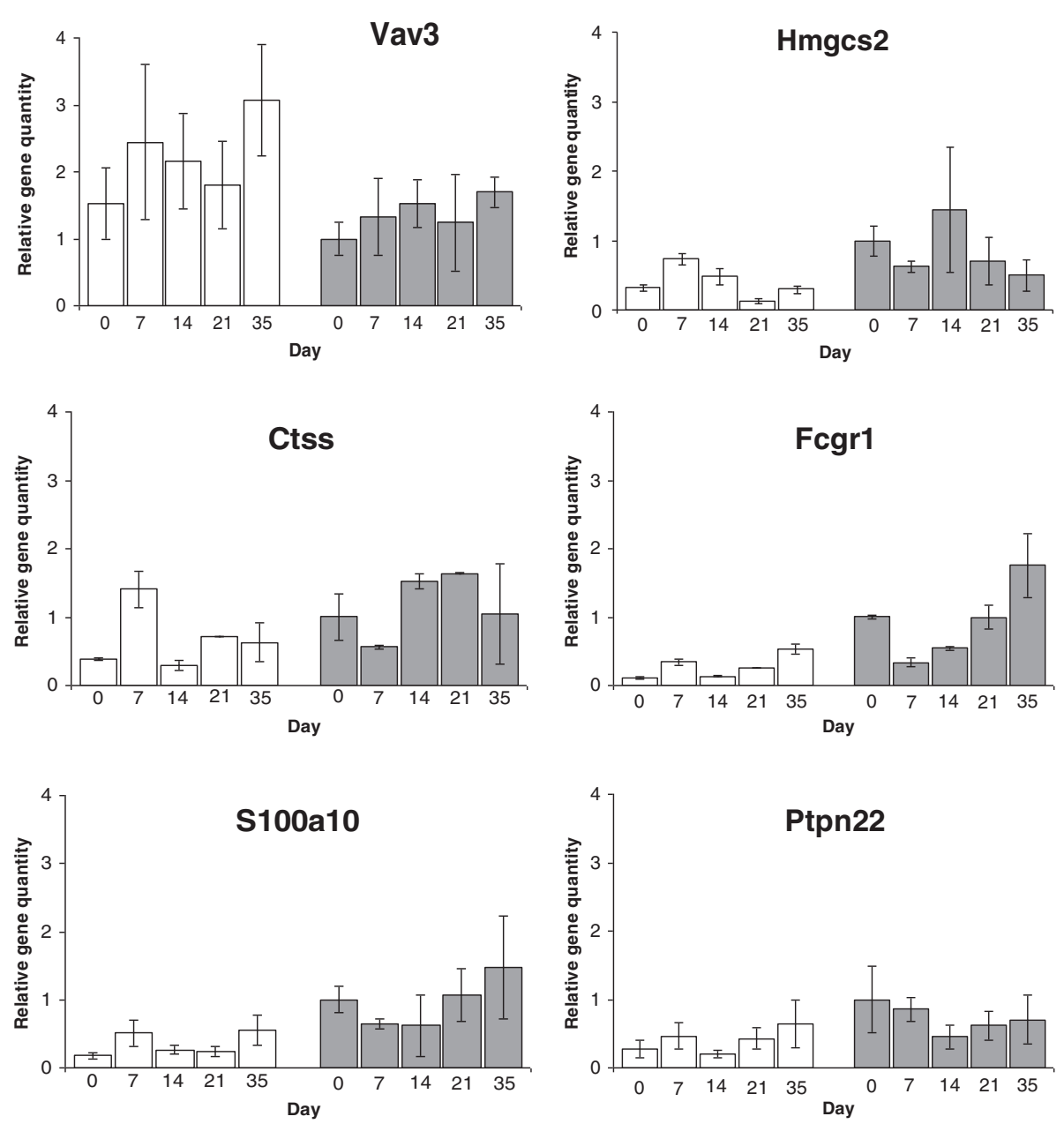

Figure 4 Colonic Tm3 gene expression by independent qPCR. Results are displayed relative to naïve resistant BALB/C, following standardization and normalization of samples against housekeeper gene ( $\beta$-actin). Shown are the top 3 candidates by pathway \& SNP analysis (Table 1: Vav3, Hmgcs2 \& CTSS), the current strongest candidate gene from the literature (Fcgr1), the candidate with the highest text mining score but no designated biological pathway (Table 3: S100a10) and the candidate with the most SNPs but as yet without a designated pathway (Table 3: Ptpn22). Open bars denote susceptible AKR, shaded bars denote resistant BALB/c.

with highly similar pathology to both the IL10 deficient and T. muris models of colonic inflammation [9,24]. The remaining 2 genes are highly polymorphic and known to be important in inflammation (RORc, Ptpn22). As individual candidate genes, each demonstrates interesting biological functionality that could play a role in mucosal inflammation. For instance, FcgR1 codes for a high affinity IgG receptor, key to IgG2a-induced phagocytosis and antigen specific immune responses. In the mouse, FcgR1 has been associated with autoimmune disorders such as rheumatoid arthritis and bacterial infection [25]. In humans the closely related $F c g R 2 a$ and $F c g R 3$ have been associated with IBD [26]. The protein tyrosine phosphatase gene (Ptpn22) is of particular interest, as in humans a mis-sense SNP (C1858T) has already demonstrated strong correlation with rheumatoid arthritis [27], type-1 diabetes mellitus [28], and other autoimmune disease [29].
Interestingly, the C1858T gene variant is not associated with the establishment of human Crohn's disease [30] and may even represent protection [31]. In our study, Ptpn 22 demonstrated progressively increased expression within the colonic tissue of susceptible mice following the establishment of colitis.

The unbiased approach we have used to select candidate genes has also highlighted a gene whose currently assigned pathway (circadian rhythm) does not overtly relate to mucosal inflammation. The Retinoic acid-related orphan receptor-C $(R O R c / R O R \gamma)$ gene encodes for ROR $\gamma \mathrm{t}$ (ROR 2 2), a lineage-specific transcription factor of CD4+ $\mathrm{T}_{\mathrm{H}} 17$ cell differentiation [32]. Excessive $\mathrm{T}_{\mathrm{H}} 17$ cell activity has been implicated in both autoimmune [33] and inflammatory bowel diseases [34].

Finally, Vav3 was the primary candidate revealed by integrative pathway and SNP analysis and is of particular 
interest, as in six week old $\operatorname{Vav} 1 / 2 / 3$ triple knockout mice altered gut enterocyte differentiation and morphology has been shown, along with spontaneous colitis and ulceration in the caecum and ascending colon [35]. Vav3 is also involved in at least 7 known biological pathways, all of which could play a role in mucosal homeostasis and regulation. Some of these pathways involve other candidate genes in this region, for instance FcgR1 (Fc-gamma receptor mediated phagocytosis), Vcam1 (leukocyte transendothelial migration, focal adhesion) and Ptpn22 (negative regulation of T-cell receptor signalling) [36,37]. We hypothesise therefore that Cdcs1 is in fact a 'colitis hotspot' containing several genes which if dysregulated through genetic variation, could adversely affect gut inflammation. It is possible that the specific candidate genes for each colitis model are not the same. However, the biological interaction between genes at this locus, demonstrates the importance of $C d c s 1$ and why this region appears in unrelated models of gut inflammation.

Interestingly, $\operatorname{Tm} 3$ (Cdcs1) does not correlate with any known nematode infection susceptibility QTL, but instead appears exclusive to colonic inflammatory disease.

For instance, expulsion and resistance to the small intestinal nematode Heligmosomoides bakeri in mice has been characterized at murine chromosome 1 and 17 [38] corresponding to $T m 1$ and Tm17. Similarly, a study of Trichinella spiralis infection in rats, which causes acute and transient small bowel inflammation, identified a single significant QTL region homologous to the murine chromosome 1 locus (Tm1) [39]. Lastly, resistance to small bowel and abomasum/gastric nematode infections of sheep, have highlighted a number of suggestive QTL [40-42], homologous to Tm17, and downstream of Tm10. All studies demonstrated that resistance/susceptibility to GI nematode infection is under multi-genetic control, with MHC and non-MHC loci important in outcome [43]. However, these studies also highlight the importance of the $C d c s 1$ locus with the establishment of a large bowel inflammatory phenotype, separate to precise anti-parasitic mechanisms.

In conclusion, we have corroborated three previously published studies which associate the locus $C d c s 1$ with colonic mucosal inflammation in the mouse. Furthermore, we have shown that in the AKR and BALB/c, genetic variation in this region has the potential to affect mucosal homeostasis through several different pathways. Most importantly, we have demonstrated that an unbiased integrative analysis can be beneficial in candidate gene identification and prioritization, particularly cisregulated genes, even in large regions. This approach is particularly useful for hypothesis generation, and has positionally implicated Vav3 as a biologically relevant gene candidate in colitis.

\section{Methods}

\section{Animals}

Mice were housed with free access to food and water under specific pathogen free conditions. All experiments were performed under regulations of The UK Home Office Animals (Scientific Procedures) Act of 1986.

For QTL analysis, AKR/OlaHsd (susceptible, hereafter referred to AKR) and BALB/cOlaHsd (resistant, hereafter referred to $\mathrm{BALB} / \mathrm{c}$ ) mice (Harlan Olac, UK) were interbred. To generate an F1 population of mice, equal numbers of AKR males vs BALB/c females (F1a offspring), and $A K R$ females vs BALB/c males (F1b offspring) were mated. At least 50 breeding-pairs of F1-mice were then interbred. All F1 vs F1 breeding was performed over the same time-period. To maintain genetic balance, F1a males were bred with F1a and F1b females; and, F1b males with F1a and F1b females. A single generation of 307 F2 mice (male and female) was created for study. All F2 mice were infected at the same time with $T$. muris ova at 6-8 weeks of age.

\section{Parasites}

Trichuris muris parasites were harvested and ova collected and maintained as previously described [18]. All infected mice received 300 T. muris ova in distilled water $(200 \mu \mathrm{l})$ by oral gavage.

\section{QTL phenotyping}

Phenotypic analysis was performed for all 307 F2 mice. Day 35 post-infection, serum samples and intestines were taken at autopsy. Resistance (0 worm load) and susceptibility ( $>0$ worms) were defined. All worm counts were performed by a single investigator over 1 week from caeca frozen at autopsy. This method of storage and counting is used routinely for large experiments and does not affect quantification. Parasite-specific antibody ELISA was performed as described previously [44], using in-house T. muris excretory-secretory (ES) protein. T. muris specific IgG1 ( $\mathrm{T}_{\mathrm{H}} 2$ specific, driven by IL4) and IgG2a ( $\mathrm{T}_{\mathrm{H}} 1$ specific, driven by IFN $\gamma$ ) optical density (OD) was measured simultaneously for all samples. All 307 serum ELISAs were performed in one run. For histology, $0.5 \mathrm{~cm}$ of whole colonic segments from the proximal ascending colon was snap-frozen, thawed in $4 \%$ paraformaldehyde, paraffin embedded, and $5 \mu \mathrm{m}$ transverse tissue sections stained with Haematoxylin and Eosin (H\&E) simultaneously. 50 randomly assigned colonic specimens were assessed. Proximal colonic specimens were scored according to colonic crypt length $(\mu \mathrm{m})$, immune cell infiltration and tissue inflammation by a single investigator. Colonic crypt length per individual was taken as a mean across at least 20 crypt units and 3 separate sections. Crypt units were measured using Image-J software [45]. Spearman's rank correlation 
coefficient was performed to measure the statistical dependence of (a) worm count and colonic crypt length variables, and (b) IgG1:IgG2a ratio and crypt length variables.

\section{DNA extraction}

DNA was isolated (Promega Wizard DNA isolation kit) from tail snips digested in proteinase $\mathrm{K}$ digestion buffer (20 $\mathrm{mg} / \mathrm{ml}$ ). DNA concentration was determined by Nanodrop spectrophotometer and then stored at $-80^{\circ} \mathrm{C}$ until analysis.

\section{Linkage Map}

165 polymorphic murine microsatellite markers distinguishing between AKR and BALB/c were selected [46]. Whole genome coverage was $85 \%$ and median intermarker distance $12.3 \mathrm{cM}$. Conversion of marker positions from recombination fraction (cM) to physical position (Mb) was achieved using the Ensembl database [47].

\section{Microsatellite amplification and genotype analysis}

Forward polymerase chain reaction (PCR) primers were fluorescently labelled with 6-FAM, HEX or NED (MWG Biotec, Applied Biosystems). 25 ng of genomic DNA was used for each marker. Semi-automated analysis of genotypes on pooled panels of PCR products was performed using an Applied Biosystems 3100 Capillary sequencer with Genescan analysis and Genotyper software.

\section{QTL analysis}

IgG1:IgG2a ratios were $\log _{10}$ transformed to achieve parametric distribution. Median, mean and kurtosis values were calculated using QStat, Windows QTL Cartographer 2.5. Normalised data were analysed using multiple interval mapping to optimize and refine QTL positions. A genome-wide permutation test (1000 repeats) determined thresholds for significance; a logarithm of odds (LOD) score of 4.0 or a $\mathrm{p}$ value of $<5.2 \times 10^{-5}$ was considered significant. A LOD score of 2.5 or $\mathrm{p}$ value of $1.6 \times 10^{-3}$ was considered suggestive of linkage according to published guidelines [48]. All significant LOD scores were confirmed by 1-way ANOVA with pairwise comparison, using the Bonferroni correction method. Kruskal-Wallis analysis was used for worm burden and IgG2a data, and converted to an LOD score [49].

\section{Genome-wide colonic transcriptional activity of parental murine strains}

Naïve and infected 6-to-8 week old male AKR and $\mathrm{BALB} / \mathrm{c}$ mice (Harlan Olac, UK) were monitored through to day 35 post-infection $(n=6,3$ experimental replicates for each conditional cohort) as described previously [9]. 3 replicate pooled samples of colonic RNA (ascending colon) were generated for each experimental group.
Whole transcriptome microarray expression analysis (Affymetrix Genechip Mouse Exon 1.0 ST Array ${ }^{\circledR}$ ) and bioinformatic analysis was performed. The entire genomewide expression dataset was used for subsequent analysis $[9,50]$.

\section{The in-silico prioritization of QTL candidate genes}

The use of workflows in the analysis of large-scale genomic data provides a systematic and un-biased mechanism for hypothesis generation [51]. Previously constructed workflows were re-used for the analysis of QTL and gene expression data, to identify biological pathways which correlated with Trichuris muris infection. The identification of candidate genes underlying each QTL was carried out by firstly determining the precise co-ordinates of each genetic marker (Mbp) (Table 1). Each QTL was subsequently entered into the workflow qtl_to_pathway (Additional file 1: Figure S2 [16]). Genes located within each QTL were annotated with additional accession number identifiers (including UniProt ID and Entrez Gene IDs), in order to cross-reference Ensembl database identifiers to KEGG (Kyoto Encyclopaedia of Genes and Genomes) [52] pathway identifiers. As a result, annotated biological pathways were extracted from the KEGG database for inclusion in further analysis.

In parallel, differentially expressed genes identified from the $T$. muris microarray study [9] were analysed using the refseq_ids_to_pathways workflow (Additional file 1: Figure S3 [17]). This workflow required preliminary analysis of the gene expression data [9] (Partek Genomic Solution version 6.5, 2009, Partek, USA) and conversion of Affymetrix probe-set identification markers to their recognised NCBI RefSeq identification code (refseq ids). An identical process to that of the qtl_to_pathway workflow for gene annotation was then carried out.

The mapping of gene expression data to KEGG highlighted biological pathway activity in the pathogenesis of colonic disease. All genes with significant transcriptional differences between resistant and susceptible strains, in naïve and infected states (ANOVA, factor interaction, $\mathrm{p}<0.05$ ), were included for analysis. To identify cis-QTL genes of biological relevance to phenotype, those genes with a higher degree of over/under expression (Fold Change $\geq+/-1.4$ over naïve levels) during chronic $T$. muris intestinal inflammation, were used in the workflow analysis (see Figure 3).

The workflow common_pathways (Additional file 1: Figure S4 [53]) was used to identify candidate pathways containing differentially expressed genes within a QTL, in order to obtain an overall view of the mechanisms which may be influencing the expression of the phenotype.

Additional text mining was used to prevent potential candidate genes which lacked KEGG pathway annotation from being discarded. Transcribed QTL genes were analysed 
using a text mining workflow (Additional file 1: Figure S5 [54]). Briefly, published abstracts were identified from a PubMed search using the term "("Colitis" AND "Inflammation") AND ("Human" OR "Mouse")". All scientifically relevant keywords contained within individual abstracts were extracted, constructing a phenotype concept profile and allowing the calculation of inverse document frequency (IDF) scores ie a score relating the number of resulting documents which contained the keywords in question. In parallel, abstracts pertaining to selected genes were similarly recorded. The identification of phenotype keywords within individual gene abstracts allowed for the generation of a cosine vector score for each gene ranging from +1 to $-1(+1=$ causation of phenotype; $0=$ unknown association with phenotype; $-1=$ preventative of phenotype). Ranked by their cosine vector score, the association with phenotype of a particular gene was displayed. Similarly, individual phenotype keywords were also ranked according to the IDF scores, identifying possible correlations between each gene and the phenotype. All data regarding text mining and workflow approaches are published online [55].

Only QTL genes known to possess SNP variation between parental AKR and BALB/c [56] were subject to further analysis.

\section{Independent replication of candidate gene expression by qPCR (Tm3)}

Infected parental strains AKR and BALB/c (Harlan Olac, UK) received $300 T$. muris ova by oral gavage. Mice were culled days 0 (naive), 7, 14, 21 and 35 post-infection for analysis ( $\mathrm{n}=3$ for each cohort). mRNA was extracted from $0.5 \mathrm{~cm}$ of whole colonic tissue segments, from the ascending colon, according to manufacturer's instruction (TRIZOL $^{\circledR}$, Invitrogen). cDNA was synthesised. A full list of gene primers (Eurofins-MWG-Operon, Germany) and their sequences are provided (Additional file 1: Table S1). Samples were quantitatively analysed using KAPA SYBR FAST qPCR Master Mix (Kapa Biosystems Inc., USA) and a Bio-Rad MyIQ ${ }^{\mathrm{Tm}}$ PCR detection system (Bio-Rad IQ5 optical system software, version 2; Bio-Rad Laboratories Inc., ( )). Three replicate cDNA samples were run at a 1:20, a 1:100, and a 1:500 dilutions for each time-point. Threshold cycles were calculated; gene detection within the three serially diluted samples was standardized, and then normalized against housekeeping gene beta-actin (Act-b) . Relative fold change in gene quantity was calculated using naïve resistant mice as a reference.

\section{Additional file}

Additional file 1: Contains Supplementary Figure S1 showing gender variation in phenotype. Also Supplementary Figures S2-S5 which detail stepwise representations of workflows mentioned in
Methods (see legends below), and Table S1 showing primer sequences for qPCR. Table S1: Primer sequences for qPCR-amplified genes. Figure S1: Phenotype data for whole cohort, stratified by gender. A: Females showed significantly lower worm burden compared to males (Mann Whitney $U$ test, $p<0.0001)$. B: Females showed significantly higher $\operatorname{lgG} 1: 2 \mathrm{a}$ antibody ratio ( $T$ test, $\mathrm{p}<0.0001)$. Figure $\mathbf{S 2}$ : Pathways and Gene annotations for QTL region. This workflow searches for genes which reside in a QTL (Quantitative Trait Loci) region in the mouse, Mus musculus. The workflow requires an input of: a chromosome name or number; a QTL start base pair position; QTL end base pair position. Data is then extracted from BioMart to annotate each of the genes found in this region. The Entrez and UniProt identifiers are then sent to KEGG to obtain KEGG gene identifiers. The KEGG gene identifiers are then used to search for pathways in the KEGG pathway database. (http://www. myexperiment.org/workflows/1661.html). Figure S3: Pathways and Gene annotations for RefSeq ids. This workflow searches for Mus musculus genes found to be differentially expressed in a microarray study. The workflow requires an input of gene ref_seq identifiers. Data is then extracted from BioMart to annotate each of the genes found for each ref_seq id. The Entrez and UniProt identifiers are then sent to KEGG to obtain KEGG gene identifiers. The KEGG gene identifiers are then used to search for pathways in the KEGG pathway database. (http://www. myexperiment.org/workflows/1662.html). Figure S4: KEGG pathways common to both QTL and microarray based investigations. This workflow takes in two lists of KEGG pathway ids. These are designed to come from pathways found from genes in a QTL (Quantitative Trait Loci) region, and from pathways found from genes differentially expressed in a microarray study. By identifying the intersecting pathways from both studies, a more informative picture is obtained of the candidate processes involved in the expression of a phenotype. (http://www.myexperiment.org/ workflows/1663.html). Figure S5: Pathway and Gene to Pubmed. This workflow takes in a list of gene names, KEGG pathway descriptions and phenotypes as keywords, and searches the PubMed database for corresponding articles. Retrieved abstracts are then used to calculate a cosine vector space between two sets of corpora (gene and phenotype, or pathway and phenotype). The workflow counts the number of articles in the PubMed database in which each term occurs, and identifies the total number of articles in the entire PubMed database so that a term enrichment score may be calculated. Scientiifc terms are then extracted from the abstract text and given a weighting according to the number of terms that appear in the document. The higher the value the better the score. This is given as: $X($ or $Y)=\log ((a / b) /(c / d))$ where: $a=$ number of occurrences of individual terms in phenotype (or pathway) corpus, $b=$ number of abstracts in entire phenotype (or pathway) corpus, $c=$ number of occurrences of individual terms in entire PubMed,

$d=$ number of articles in entire PubMed. Once this has been created, the pathways obtained from the QTL and microarray pathway analysis workflows are analysed. The weighted terms are then given a link score $X+Y$. The higher the score the more "appropriate/interesting" the link between the pathway and the phenotype. This is calculated as: $W=(X+Y)$. (http://www.myexperiment.org/workflows/1846.html).

Competing interests

The author declares that they have no competing interests.

\section{Authors' contributions}

SL: Study design; data acquisition; data analysis; data interpretation drafting and writing of manuscript; obtaining funding. PF: Data analysis concept and design; data acquisition; data analysis; data interpretation; writing of manuscript. JH: Data acquisition; technical support. LZ: Data analysis; bioinformatics. SE: Data analysis; data interpretation. WO: Data analysis; data interpretation. JM: Revision of manuscript. AB: Data analysis concept and design; data interpretation. RG: Study concept and design; obtaining funding; study supervision. JP: Study concept and design; data acquisition; data analysis; data interpretation; drafting and critical revision of manuscript; study supervision. All authors read and approved the final manuscript.

Authors' information

Richard K Grencis and Joanne L Pennock co-senior author. 


\section{Acknowledgements}

The authors acknowledge the Wellcome Trust (RKG, JLP) and MRC (SL Clinical Fellowship) for funding this work. Also the staff in the BSF of the University of Manchester for technical assistance and support.

\begin{abstract}
Author details
${ }^{1}$ Gastrointestinal Sciences, Institute of Inflammation and Repair, Faculty of Medicine and Human Sciences, University of Manchester, 4.004 AV Hill Building, Oxford Road, Manchester M13 9PT, UK. 'Bioinformatics Scientist, Oncology, AstraZeneca, Cheshire, UK. ${ }^{3}$ Centre for Integrated Genomic Medical Research, Institute of Population Health, Faculty of Medicine and Human Sciences, University of Manchester, Manchester, UK. ${ }^{4}$ Bioinformatics, Faculty of Life Sciences, University of Manchester, Manchester, UK. ${ }^{5}$ Arthritis Research UK Epidemiology Unit, Institute of Inflammation and Repair, Faculty of Medicine and Human Sciences, University of Manchester, Manchester, UK. ${ }^{6}$ School of Computer Sciences, University of Manchester, Manchester, UK.

${ }^{7}$ Faculty of Life Sciences, University of Manchester, Manchester, UK.
\end{abstract}

Received: 30 October 2012 Accepted: 14 February 2013

Published: 26 February 2013

\section{References}

1. Wellcome Trust Case Control Consortium: Genome-wide association study of 14,000 cases of seven common diseases and 3,000 shared control. Nature 2007, 447:661-678.

2. McCarthy MI, Abecasis GR, Cardon LR, Goldstein DB, Little J, Ionnidis JP, Hirschhorn JN: Genome-wide association studies for complex traits: consensus, uncertainty and challenges. Nat Rev Genet 2008, 9:356-369.

3. Glazier AM, Nadeau JH, Altman TJ: Finding genes that underlie complex traits. Science 2002, 298:2345-2349.

4. Stylianou IM, Affouritt JP, Shockley KR, Wilpan RY, Abdi FA, Bhardwaj S, Rollins J, Churchill GA, Paigen B: Applying gene expression, proteomics and single-nucleotide polymorphism analysis for complex trait gene identification. Genetics 2008, 178:1795-1805

5. Olofsson P, Holmberg J, Tordsson J, Lu S, Akerstrom B, Holmdahl R: Positional identification of $\mathrm{Ncf} 1$ as a gene that regulates arthritis severity in rats. Nat Genet 2003, 33:25-32

6. Cliffe $L$, Grencis RK: The Trichuris muris system: a paradigm of resistance and susceptibility to intestinal nematode infection. Adv Parasitol 2004 57:255-307.

7. Bancroft AJ, McKenzie AN, Grencis RK: A critical role for IL-13 in resistance to intestinal nematode infection. J Immunol 1998, 160:3463-3461.

8. Else KJ, Finkelman FD, Malizewski CR, Grencis RK: Cytokine mediated regulation of chronic intestinal helminth infection. J Exp Med 1994, 179:347-351.

9. Levison SE, McLaughlin JT, Zeef LA, Fisher P, Grencis RK, Pennock JL Colonic transcriptional profiling in resistance and susceptibility to trichuriasis: phenotyping a chronic colitis and lessons for iatrogenic helminthosis. Inflamm Bowel Dis 2010, 16:2065-2079.

10. Awasthi S, Bundy DA, Savioli L: Helminthic infections. BMJ 2003, 327:431-433.

11. Borm ME, He J, Kelsall B, Pena AS, Strober W, Bouma G: A major quantitative trait locus on mouse chromosome 3 is involved in disease susceptibility in different colitis models. Gastroenterology 2005, 128:74-85.

12. Farmer MA, Sundberg JP, Bristol IJ, Churchill GA, Elson CO, Leiter EH: A major quantitative locus on chromosome 3 controls colitis severity in IL10-deficient mice. Proc Natl Acad Sci USA 2001, 98:13820-13825.

13. Ermann J, Garrett WS, Kuchroo J, Rorida K, Glickman JN, BLeich A, Glimcher $\mathrm{LH}$ : Severity of innate immune-mediated colitis is controlled by the cytokine deficiency-induced colitis susceptibility-1 (Cdcs1) locus. Proc Natl Acad Sci U S A 2011, 108:7137-7141.

14. Bleich A, Buchler G, Beckwith J, Petell LM, Affourtit JP, King BL, Shaffer DJ, Roopenian DC, Hedrich HJ, Sundberg JP, Leiter EH: Cdcs1 a major colitis susceptibility locus in mice; subcongenic analysis reveals genetic complexity. Inflamm Bowel Dis 2010, 16:765-775.

15. Beckwith J, Cong Y, Sunderberg JP, et al: Cdcs1, a major colitogenic locus in mice, regulates innate and adaptive immune responses to enteric bacterial antigens. Gastroenterology 2005, 129:1473-1484.

16. Fisher P: Pathways and Gene annotations for QTL region. http://www. myexperment.org/workflows/1661.html.

17. Fisher P: Pathways and Gene annotations for RefSeq ids. http://www. myexperiment.org/workflows/1662.html.
18. Wakelin D: Acquired immunity to Trichuris muris in the albino laboratory mouse. Parasitology 1967, 57:515-524.

19. Artis D, Grencis RK: The intestinal epithelium: sensors to effectors in nematode infection. Mucosal Immunol 2008, 1:252-264.

20. Kowaiwa K, Sugawara K, Smith MF Jr, Carl V, Yamschikov V, Belyea B, McEwen SB, Moskaluk CA, Pizarro TT, Cominelli F, McDuffie M: Identification of a quantitative trait locus for ileitis in a spontaneous mouse model of crohn's disease: SAMP/YitFc. Gastroenterology 2003, 125:477-490.

21. Flint J, Valdar W, Shifman S, Mott R: Strategies for mapping and cloning quantitative trait genes in rodent. Nat Rev Genetics 2005, 6:271-286.

22. Mouse Phenome Database. http://www.informatics.jax.org.

23. Verdugo RA, Farber $\mathrm{CR}$, Warden $\mathrm{CH}$, Medrano JF: Serious limitations of the QTL/Microarray approach for QTL gene discovery. BMC Biol 2010, 8:96.

24. Bristol IJ, Farmer MA, Cong Y, Zheng XX, Strom TB, Elson CO, Sundberg JP, Leiter EH: Heritable susceptibility for colitis in mice induced by IL-10 deficiency. Inflamm Bowel Dis 2000, 6:290-302.

25. laon-Facsinay A, de Kimpe SJ, van Lent PL, Hofhuis FM, Van Ojik HH, Sedlik C, da Silveira SA, Gerver J, de Jong YF, Roozendaal R, Aarden LA, van den Berg WB, Saito T, Mosser D, Amigorena S, Izui S, van Ommen GJ, van Vugt $M$, van de Winkel JG, Verbeek JS: Fc-gamma-R1 (CD64) contributes substantially to severity of arthritis, hypersensitivity responses, and protection from bacterial infection. Immunity 2002, 16:391-402.

26. Weersma RK, Crusius JB, Roberts RL, Koeleman BP, Palomino-Morales $\mathrm{R}$ Wolkamp S, Hollis-Moffatt JE, Festen EA, Meisneris S, Heijmans R, Noble CL, Gearry RB, Barclay ML, Gomez-Garcia M, Lopez-Nevot MA, Nieto A, Rodrigo L, Radstake TR, van Bodegraven AA, Wijmenga C, Merriman TR, Stokkers PC, Pena AS, Martin J, Alizadeh BZ: Association of FcgR2a, but not FcgR3a, with inflammatory bowel disease across three Caucasian populations. Inflamm Bowel Dis 2010, 16:2080-2089.

27. Begovich AB, Carlton VE, Honigberg LA, Schrodi SJ, CHokkalingam AP, Alexander HC, Ardlie KG, Huang Q, Smith AM, Spoerke JM, Conn MT, Chang M, Chang SY, Siki RK, Catanese JJ, Leong DU, Garcia VE, MCAllister LB, Jeffery DA, Lee AT, Batliwalla F, Remmers E, Criswell LA, Seldin MF, Kastner DL, Amos Cl, Sninsky JJ, Gregersen PK: A missense single-nucleotide polymorphism in a gene encoding a protein tyrosine phosphatase (PTPN22) is associated with rheumatoid arthritis. Am J Hum Genet 2004 75:330-337.

28. Bottini N, Musumeci L, Alonso A, Rahmouni S, Nika K, Rostamkhani M, MacMurray J, Meloni GH, Lucarelli P, Pellecchia M, Eisenbarth GS, Comings $D$, Mustelin T: A functional variant in lymphoid tyrosine phosphatase is associated with type I diabetes. Nat Genet 2004, 36:337-338.

29. Lee YH, Rho YH, Choi SJ, Ji JD, Song GG, Nath SK, Harley JB: The Ptpn22 C1858T functional polymorphism and autoimmune disease - a metaanalysis. Rheumatology 2007, 46:49-56.

30. van Oene M, Wintle RF, Lui X, Yazdanpanah M, Gu X, Newman B, Kwan A, Johnsn B, Owen J, Greer W, Mosher D, Maksymowych W, Keystone E, Rubin LA, Amos Cl, Siminovitch KA: Association of the lymphoid tyrosine phosphatase R620W variant with rheumatoid arthritis, but not Crohn's disease, in Canadian populations. Arthritis Rheum 2005, 52:1993-1998.

31. Barrett JC, Hansoul S, Nicolae DL, Cho JH, Duerr R, Rioux JD, Brant SR, Silverberg MS, Raylor D, Barmada MM, Bitton A, Dasopoulos T, Datta LW, Green T, Riffiths AM, Kistner EO, Murtha MT, Reguieiro MD, Rotter Jl, Schumm LP, Steinhart AH, Targan SR, Xavier RJ, NIHHKIBD Genetics Consortium, Libioulle C, Sandor C, Lthrop M, Belaiche J, Dewit O, Gut I, et al: Genome-wide association defines more than 30 distinct susceptibility loci for Crohn's disease. Nat Genet 2008, 40:955-962.

32. Ivanov I, McKenzie BS, Zhou L, Tadokoro CE, Lepelley A, Lafaille JJ, Cua DJ, Littman DR: The orphan nuclear receptor RORyt directs the differentiation program of proinflammatory $\mathrm{IL}-17^{+} \mathrm{T}$-helper cells. Cell 2006, 126:1121-1133.

33. Martinez GJ, Nurieva Rl, Yang $X O$, Dong $C$ : Regulation and function of proinflammatory TH17 cells. Ann N Y Acad Sci 2008, 1143:188-211.

34. Sarra M, Pallone F, MacDonald TT, Monteleone G: I23/IL17 axis in IBD. Inflamm Bowel Dis 2010, 16:1808-1013.

35. Liu JY, Seno H, Miletic AV, Mills JC, Swat W, Stappenbeck TS: Vav proteins are necessary for correct differentiation of mouse caecal and colonic enterocytes. J Cell Sci 2009, 122:324-334.

36. Zeng L, Sachdev P, Yan L, Chan JL, Trengle T, McClelland M, Welsh J, Wang $\mathrm{LH}$ : Vav3 mediates receptor protein tyrosine kinase signalling, regulates GTPase activity, modulates cell morphology and induces cell transformation. Mol Cell Biol 2000, 20:9212-9224. 
37. Tybulewicz VLJ: Vav-family proteins in T-cell signalling. Curr Opin Immunol 2005, 17:267-274.

38. Behnke JM, Iraqi FA, Mugambi JM, Clifford S, Nagda S, Wakelin D, Kemp SJ, Baker RL, Gibson JP: High resolution mapping of chromosomal regions controlling resistance to gastrointestinal nematode infections in an advanced intercross line of mice. Mamm Genome 2006, 17:584-597.

39. Suzuki T, Ishih A, Kino H, Muregi FW, Takabayashi S, Nishikawa T, Takagi H, Terada M: Chromosomal mapping of host resistance to Trichinella spiralis nematode infection in rats. Immunogenetics 2006, 58:26-30.

40. Beh KJ, Hulme DJ, Callaghan MJ, Leish Z, Lenane I, Windon RG, Maddox JF: A genome scan for quantitative trait loci affecting resistance to Trichostrongylus colubriformis in sheep. Anim Genet 2002, 33:97-106.

41. Dominik S: Quantitative trait loci for internal nematode resistance in sheep: a review. Genet Sel Evol 2005, 37(Suppl.1):S83-S96.

42. Stear MJ, Boag B, Cattadori I, Murphy L: Genetic variation in resistance to mixed, predominantly Teladorsagia circumcincta nematode infection of sheep: from heritabilities to gene identification. Parasite Immunol 2009, 31:274-282

43. Behnke JM, Menge DM, Noyes H: Heligmosomoides bakeri: a model for exploring the biology and genetics of resistance to chronic gastrointestinal nematode infections. Parasitology 2009, 136:1565-1580.

44. Else KJ, Entwistle GM, Grencis RK: Correlation between worm burden and markers of Th1 and Th2 cell subset induction in an inbred strain of mouse infected with Trichuris muris. Parasite Immunol 1993, 15:595-600.

45. Image-J Software. http://rsbweb.nih.gov/ij.

46. Broad Institute. http://www.broad.mit.edu/mouse.

47. Ensembl Database: Ensembl Database. http://www.ensembl.org] Ensembl 64 assembly accessed September 2011.

48. Lander E, Kruglyak L: Genetic dissection of complex traits: guidelines for interpreting and reporting linkage results. Nat Genet 1995, 11:241-247.

49. Broman KW: Mapping quantitative trait loci in the case of a spike in the phenotype distribution. Genetics 2003, 163:1169-1175.

50. Genome-wide array data: Genome-wide array data. available at: [http:// www.ebi.ac.uk/arrayexpress/experiments/E-MEXP-3098].

51. Fisher P, Hedeler C, Wolstencroft K, Hulme H, Noyes H, Kemp S, Stevens R, Brass A: A systematic strategy for large-scale analysis of genotypephenotype correlations: identification of candidate genes involved in African trypanosomiasis. Nucleic Acids Res 2007, 35:5625-5633.

52. Kanehisa M, Goto S: KEGG: Kyoto encyclopaedia of genes and genome. Nucleic Acid Res 2000, 28:27-30

53. Fisher P: KEGG pathways common to both QTL and microarray based investigations. http://www.myexperiment.org/workflows/1663.

54. Fisher P: Pathway and Gene to PubMed; a text mining workflow. http:// www.myexperiment.org/workflows/1846.

55. Fisher P: http://www.myexperiment.org/packs/169.

56. Sanger Mouse SNP Repository. http://www.sanger.ac.uk/cgi-bin/ modelorgs/mousegenomes/snps/pl.

doi:10.1186/1471-2164-14-127

Cite this article as: Levison et al: Genetic analysis of the Trichuris murisinduced model of colitis reveals QTL overlap and a novel gene cluster for establishing colonic inflammation. BMC Genomics 2013 14:127.

\section{Submit your next manuscript to BioMed Central and take full advantage of:}

- Convenient online submission

- Thorough peer review

- No space constraints or color figure charges

- Immediate publication on acceptance

- Inclusion in PubMed, CAS, Scopus and Google Scholar

- Research which is freely available for redistribution 